\title{
INFLUÊNCIA DA TEMPERATURA E ATMOSFERA DE SECAGEM SOBRE A RESISTÊNCIA MECÂNICA DE UM AGLOMERADO DE MINÉRIO DE FERRO PARA USO EM SINTERIZAÇÃO*
}

\author{
Flávio de Castro Dutra ${ }^{1}$ \\ Anderson Dias ${ }^{2}$ \\ Geraldo Magela da Costa ${ }^{3}$ \\ Marcus Emrich ${ }^{4}$
}

\section{Resumo}

Este artigo apresenta a influência da temperatura e da atmosfera de secagem sobre a resistência mecânica de um aglomerado esférico, com diâmetro entre $3 \mathrm{~mm}$ e 8 $\mathrm{mm}$, constituído de pellet feed e silicato de sódio, produzido através de pelotamento em discos e endurecido em temperaturas até $550^{\circ} \mathrm{C}$. O aglomerado obtido a partir deste processo apresenta características que permitem que o mesmo possa ser transportado a longas distâncias e manuseado até a sua utilização na sinterização. As principais técnicas experimentais utilizadas neste estudo foram difração de raios$\mathrm{X}$, espectroscopia Raman, microscopia eletrônica de varredura e análise térmica. $\mathrm{O}$ aglomerado foi submetido à secagem em temperaturas entre $100^{\circ} \mathrm{C}$ e $550^{\circ} \mathrm{C}$ e atmosferas constituídas de ar atmosférico e/ou $\mathrm{CO}_{2}$. Durante o processo de secagem, observou-se a influência da temperatura e da atmosfera sobre a resistência à compressão do aglomerado após exposição à água, assim como na morfologia do silicato de sódio, evidenciando uma dependência entre elas. O uso de $\mathrm{CO}_{2}$ durante a secagem mostrou-se eficiente para a formação de um aglomerado com maior resistência mecânica após exposição à água.

Palavras-chave: Aglomerado de minério de ferro; Resistência mecânica; Silicato de sódio líquido.

\section{INFLUENCE OF DRYING TEMPERATURE AND ATMOSPHERE ON MECHANICAL STRENGTH OF IRON ORE AGGLOMERATE FOR USE IN SINTERING}

\section{Abstract}

This paper presents the influence of the drying temperature and atmosphere on the mechanical strength of agglomerate composed by pellet feed and sodium silicate, produced by balling and cured at temperatures up to $550^{\circ} \mathrm{C}$. The product obtained from this process has high mechanical strength that allows it to be transported over long distances and handled until its use in sintering. The main experimental techniques used in this study were X-ray diffraction, Raman spectroscopy, scanning electron microscopy and thermal analysis. The agglomerate was submitted to drying/cure at temperatures varying between 100 and $550^{\circ} \mathrm{C}$ and gaseous atmosphere consisting of air and /or carbon dioxide $\left(\mathrm{CO}_{2}\right)$. During the drying/cure processes, it was observed a strong influence of both temperature and atmosphere on the compression strength after exposure to water, as well as on the morphology of the sodium silicate, demonstrating a dependency between them. The use of $\mathrm{CO}_{2}$ during drying proved to be effective to achieve an increase of compression strength after exposure to water.

Keywords: Iron ore agglomerate; Mechanical strength; Sodium silicate.

1 Pesquisador, Centro de Tecnologia de Ferrosos, Vale S.A, Nova Lima (MG), Brasil.

2 Professor, Departamento de Química, Universidade Federal de Ouro Preto, Ouro Preto (MG), Brasil.

3 Professor, Departamento de Química, Universidade Federal de Ouro Preto, Ouro Preto (MG), Brasil.

4 Pesquisador, Centro de Tecnologia de Ferrosos, Vale S.A, Nova Lima (MG), Brasil.

* Contribuição técnica ao 44 Seminário de Redução de Minério de Ferro e Matérias-primas, $15^{\circ}$ Simpósio Brasileiro de Minério de Ferro e $2^{\circ}$ Simpósio Brasileiro de Aglomeração de Minério de Ferro, 15 a 18 de setembro de 2014, Belo Horizonte, MG, Brasil. 


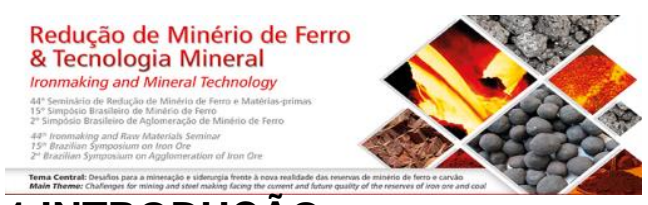

\section{INTRODUÇÃO}

Uma tecnologia de produção de um aglomerado de minério de ferro com diâmetro entre 3 e $8 \mathrm{~mm}$ foi desenvolvida por Dutra e Pimenta [1]. Esse aglomerado foi produzido a partir da mistura de pellet feed com silicato de sódio líquido. O produto obtido a partir desse processo apresentou alta resistência mecânica, permitindo a principio que o mesmo possa ser transportado a longas distâncias e manuseado até a sua utilização na sinterização. O aglomerado e a rota para sua obtenção minimizam alguns problemas normalmente encontrados nos processos de aglomeração com cura a frio e/ou a baixas temperaturas, tais como necessidade de altas dosagens de aglomerantes, alto tempo para cura do produto, baixa resistência ao contato com água, alta geração de finos por transporte e manuseio e alta geração de finos por choque térmico. Testes realizados em sinterização piloto comprovaram que o produto tem excelente desempenho, com ganhos significativos ao processo e à qualidade do sínter como, por exemplo, aumento da produtividade, redução de consumo específico de combustível e elevada resistência mecânica. Além disso, devido ao fato desse aglomerado ser produzido a partir de pellet feed com teores elevados de ferro e baixos teores de contaminantes, apresenta qualidade química superior à maioria dos sinter feeds. O processo de produção resume-se às etapas de mistura de pellet feed com silicato de sódio líquido em misturador horizontal, pelotamento em discos e secagem/cura do silicato de sódio para endurecimento do aglomerado.

A secagem/cura desse aglomerado em temperaturas inferiores a $550^{\circ} \mathrm{C}$ não confere ao silicato de sódio completa insolubilidade em água, promovendo uma fragilização da estrutura após exposição à água por longos períodos. Essa solubilidade em água do silicato de sódio poderá comprometer a qualidade física do aglomerado durante transporte e manuseio até a sua utilização final, gerando finos indesejáveis. A secagem e cura em temperaturas mais elevadas representam maior custo ao produto, provenientes de necessidades de estruturas mais robustas para a secagem (secadores) e gasto de energia.

A desidratação do silicato de sódio ocorre quando há calor suficiente para evaporar a água livre presente na solução. Assim, a desidratação do silicato de sódio pode ser realizada utilizando a convecção do ar quente (energia térmica), exposição à energia de microondas, ou reação exotérmica resultante de compostos orgânicos ou inorgânicos. De acordo com Owusu [2], durante a secagem, a água é transportada através da força capilar do interior do aglomerado para a superfície, a uma taxa igual à taxa de evaporação. A perda de água aumenta a taxa de polimerização e a de formação do gel. A aderência do gel aumenta entre $100^{\circ} \mathrm{C}$ e $300^{\circ} \mathrm{C}$, quando toda a água livre foi evaporada, exceto a água quimicamente ligada (água de cristalização). Boer et al. [3] também observaram pequenas perdas de água quimicamente ligadas a temperaturas inferiores a $300^{\circ} \mathrm{C}$ e uma desidroxilação contínua acima de $380^{\circ} \mathrm{C}$. Quando soluções de silicato de sódio são espalhadas uniformemente em superfícies, há a formação de filmes uniformes e finos em determinadas condições de secagem [4,5]. Durante a secagem os grupos hidroxilas $(\mathrm{OH})$ são removidos promovendo a condensação dos grupos silanol ( $\mathrm{Si}-\mathrm{OH})$ em grupos de siloxanos (Si-O-Si). Assim, a capacidade do gel para adsorver moléculas polares como a água é reduzida. Portanto, quanto mais os grupos hidroxila $(\mathrm{OH})$ são removidos a partir da sílica gel, mais o caráter hidrofílico torna-se hidrofóbico.

De acordo com Lucas et al. [6], a temperatura de secagem do aglomerado é um parâmetro importante para a consolidação físico-química dos filmes de silicato de

\footnotetext{
* Contribuição técnica ao $44^{\circ}$ Seminário de Redução de Minério de Ferro e Matérias-primas, 15은 Simpósio Brasileiro de Minério de Ferro e 2o Simpósio Brasileiro de Aglomeração de Minério de Ferro, 15 a 18 de setembro de 2014, Belo Horizonte, MG, Brasil.
} 
sódio, sendo que em temperaturas mais elevadas (acima de $150^{\circ} \mathrm{C}$ ) é observada uma coesão mecânica mais reforçada entre os grãos minerais e o aglomerante. A única diferença entre o processo de cura por $\mathrm{CO}_{2}$ e o processo de desidratação direta é que os produtos finais formados com a adição de $\mathrm{CO}_{2}$ são $\mathrm{Na}_{2} \mathrm{CO}_{3}$ e/ou $\mathrm{NaHCO}_{3}$ e sílica gel, enquanto que o último processo resulta em um gel de silicato de sódio desidratado. À medida que o silicato de sódio reage com o $\mathrm{CO}_{2}$, o sódio é consumido. A sílica gel resultante é responsável pelo aumento da resistência de ligação entre as partículas. De acordo com Kim et al. [7], durante o processo de desidratação física na presença de $\mathrm{CO}_{2}$, o silicato de sódio se decompõe em carbonato de sódio e sílica hidratada, conforme a equação: $\mathrm{Na}_{2} \mathrm{O} \cdot \mathrm{mSiO}_{2} \cdot \mathrm{nH}_{2} \mathrm{O}+$ $\mathrm{CO}_{2} \rightarrow \mathrm{mSiO}_{2} \cdot \mathrm{nH}_{2} \mathrm{O}+\mathrm{Na}_{2} \mathrm{CO}_{3}$

De acordo com Tognonvi et al. [8], a presença de cátions $\mathrm{Na}^{+}$no silicato de sódio permite que a água $\left(\mathrm{H}_{2} \mathrm{O}\right)$ e as hidroxilas $\left(\mathrm{OH}^{-}\right)$tenham um acesso mais direto nas ligações Si-O-Si. A maior presença desses cátions no filme de silicato de sódio permite a hidratação mais rápida na presença de água para reconstituir a sua esfera de hidratação e, assim, aumentar a solubilidade do material.

Com base no que foi exposto acima, este trabalho propõe o estudo dos fenômenos associados à secagem e cura do silicato de sódio e suas interações com as partículas de minério de ferro visando à busca de alternativas tecnológicas que permitam a redução da temperatura de secagem/cura, consequentemente reduzindo o custo final do produto.

\section{METODOLOGIA}

As matérias primas utilizadas são descritas a seguir:

$\checkmark \quad$ Pellet Feed: Finos de minério de ferro com 85\% das partículas com tamanhos inferiores a $0,15 \mathrm{~mm}$, com superfície específica blaine de $470 \mathrm{~cm}^{2} / \mathrm{g}$, densidade real de $4,95 \mathrm{~g} / \mathrm{cm}^{3}$, provenientes do Quadrilátero Ferrífero, estado de Minas Gerais, e compostos basicamente por $\mathrm{Fe}_{2} \mathrm{O}_{3}>96 \%, 2,2 \%$ de $\mathrm{SiO}_{2}, 0,5 \%$ de $\mathrm{Al}_{2} \mathrm{O}_{3}$, teores minoritários de $\mathrm{P}, \mathrm{Mn}, \mathrm{MgO}$ e $\mathrm{TiO}_{2}(<0,1 \%)$ e perda por calcinação (PPC) de 1,1\%;

$\checkmark \quad$ Silicato de sódio líquido: Solução de silicato de sódio fornecida pela $P Q$ Sílicas do Brasil, porcentagem de sólidos de $43 \%$, composta por $12,8 \%$ de $\mathrm{Na} 2 \mathrm{O}$, $30,2 \%$ de $\mathrm{SiO}_{2}$ e $57 \%$ de $\mathrm{H}_{2} \mathrm{O}$, densidade real de $1,56 \mathrm{~g} / \mathrm{cm}^{3}$ e viscosidade de 350 $\mathrm{cP}$ a $25^{\circ} \mathrm{C}$.

A partir dessas matérias-primas, foi preparada uma mistura de pellet feed com silicato de sódio líquido usando misturador intensivo de marca Eirich para completa homogeneização. A umidade alvo da mistura foi baseada na umidade necessária para produzir o aglomerado esférico via processo de pelotamento em disco $(9,5 \%$ de água).

Em disco de pelotamento, foram produzidos aglomerados com diâmetro entre 3 e $8 \mathrm{~mm}$, com densidade aparente de $3,1 \mathrm{~g} / \mathrm{cm}^{3}$. Os aglomerados foram submetidos à secagem, utilizando forno radiante (ULVAC-RIKO Modelo QH-E44VHT), em temperaturas variando entre 100 e $550^{\circ} \mathrm{C}$ em atmosferas gasosas constituídas de ar atmosférico $\left(78,1 \%\right.$ de $\mathrm{N}_{2} ; 20,9 \% \mathrm{O}_{2} ; 0,93 \%$ Ar e $\left.0,037 \% \mathrm{CO}_{2}\right)$ e gás carbônico (100\% de $\left.\mathrm{CO}_{2}\right)$.

Após secagem, os aglomerados foram avaliados de acordo com os seguintes ensaios:

$\checkmark \quad$ Expansão volumétrica pela medição das dimensões (diâmetro) de 15 aglomerados esféricos antes e após tratamento térmico através de paquímetro eletrônico;

* Contribuição técnica ao 44 Seminário de Redução de Minério de Ferro e Matérias-primas, 15o Simpósio Brasileiro de Minério de Ferro e 2o Simpósio Brasileiro de Aglomeração de Minério de Ferro, 15 a 18 de setembro de 2014, Belo Horizonte, MG, Brasil. 
Geração de trincas pela análise de trincas e pontos de fragilidade utilizando microscópios de luz refletida (Leica modelo DM LAN e DM LP e Nikon modelo Eclipse ME600) com sistema de análise de imagens com software Axiovision;

$\checkmark \quad$ Porosidade, na faixa de tamanho de poros entre 0.003 a $360 \mu \mathrm{m}$, por meio de porosímetro de mercúrio tipo AutoPore III (Micromeritics);

$\checkmark \quad$ Morfologia e microestrutura de fases por meio de microscopia eletrônica de varredura usando um microscópio MA15 (Carl Zeiss) e mapas de composição química dos elementos ( $\mathrm{Fe}, \mathrm{Al}, \mathrm{Si}, \mathrm{Na}$ ) utilizando um detector do tipo EDS (espectroscopia por dispersão de energia) ligado ao microscópio;

$\checkmark \quad$ Análises por espectroscopia Raman: obtenção de espectros com resolução de $1 \mathrm{~cm}^{-1}$ para contagens típicas de $60 \mathrm{~s}(10 \mathrm{X})$ após otimização do sinal. Os espectros foram obtidos em espectrômetro Horiba/Jobin-Yvon Labram-HR;

$\checkmark \quad$ Determinação de fases cristalinas por meio de difratometria de raios $X$ usando equipamento da Panalytical (modelo Empyrean) com tubo de Co. As fases cristalinas foram identificadas e interpretadas pelo software High Score Plus;

$\checkmark$ Análises térmicas (termogravimetria e calorimetria diferencial) foram realizadas em um equipamento Netzsch, modelo STA 409 usando cadinhos de alumina tipo-copo. Os experimentos foram conduzidos utilizando-se cerca de $30 \mathrm{mg}$ de amostra aquecidas entre $50^{\circ} \mathrm{C}$ e $1200^{\circ} \mathrm{C}$ a uma taxa de $5^{\circ} \mathrm{C} / \mathrm{min}$ usando fluxo de $\mathrm{N}_{2}(50 \mathrm{~mL} / \mathrm{min})$;

$\checkmark \quad$ Resistência à compressão sem e com exposição à água (períodos de $24 \mathrm{~h}$ e 30 dias) de 100 aglomerados esféricos com tamanho entre 4 e $6 \mathrm{~mm}$ por meio de prensa automática com sistema para determinação da carga de ruptura com célula de carga de capacidade de 50 daN/aglomerado, velocidade de compressão de 10 $\mathrm{mm} /$ minuto e sensibilidade de \pm 1 daN/aglomerado.

\section{RESULTADOS E DISCUSSÂO}

A resistência à compressão do aglomerado apresentou patamares semelhantes em função da temperatura de secagem. Os resultados em daN/aglomerado variaram na faixa 10-12. Por outro lado, o efeito da temperatura sobre a resistência à compressão do aglomerado após imersão em água foi significativo. O aglomerado, quando tratado termicamente a $150^{\circ} \mathrm{C}$, se degradou completamente após imersão em água. A Figura 1 mostra o efeito da temperatura sobre a perda de resistência do aglomerado secado a temperaturas $\geq 150^{\circ} \mathrm{C}$. Nota-se que à medida que aumentou a temperatura se reduziu a perda de resistência à compressão.

Sabe-se que acima de $150^{\circ} \mathrm{C}$ os grupos hidroxila $(\mathrm{OH})$ começam a ser removidos a partir do gel de sílica, tornando o filme de silicato de sódio com o caráter de superfície anteriormente hidrofílico para hidrofóbico. A maior resistência do aglomerado após exposição à água, quando o mesmo é exposto a temperaturas superiores a $250^{\circ} \mathrm{C}$ é atribuída à remoção dos grupos hidroxila $(\mathrm{OH})$, conforme mecanismo apresentado por Owusu [2]. Além disso, esses resultados estão de acordo com os estudos de Tognonvi et al. [9], que constataram que tratamentos térmicos a temperaturas mais baixas tendem a formar um filme com maior solubilidade em água, devido à tendência destes filmes (com maior presença de ligações iônicas devido ao sódio) reconstituírem sua esfera de hidratação destruída durante o processamento térmico.

Observou-se que a exposição do aglomerado em temperaturas de $150^{\circ} \mathrm{C}$ a $550^{\circ} \mathrm{C}$ em atmosfera com ar atmosférico não promove alterações volumétricas significativas. A pequena contração (1-5\%) ocorrida em praticamente todas as faixas

\footnotetext{
* Contribuição técnica ao $44^{\circ}$ Seminário de Redução de Minério de Ferro e Matérias-primas, 15은 Simpósio Brasileiro de Minério de Ferro e 2o Simpósio Brasileiro de Aglomeração de Minério de Ferro, 15 a 18 de setembro de 2014, Belo Horizonte, MG, Brasil.
} 
de temperatura reflete a perda de volume devido à perda das hidroxilas presente da goethita e no silicato de sódio. Não foram notadas formações e propagações de trincas durante aquecimento em na presença de ar atmosférico até $550^{\circ} \mathrm{C}$, fato que confere ao aglomerado integridade durante o processo de secagem.

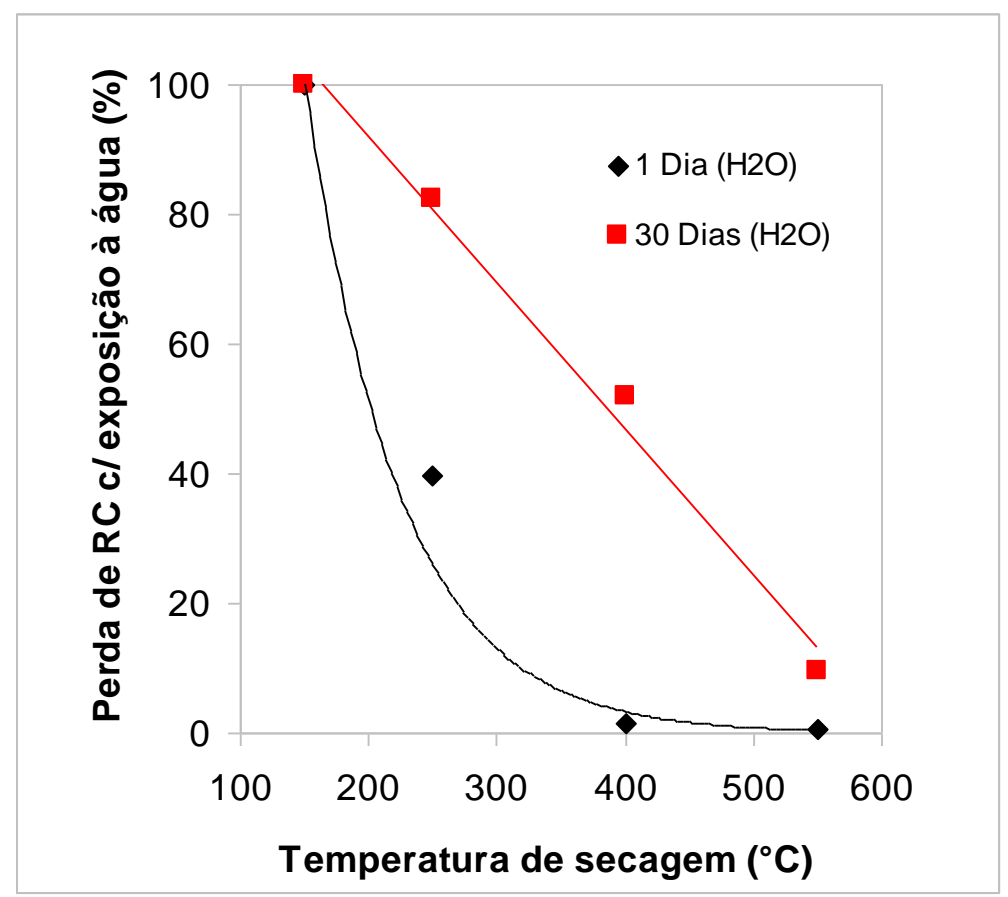

Figura 1. Efeito da temperatura sobre a resistência a compressão (RC) do aglomerado com exposição à água durante 1 e 30 dias.

No entanto, do ponto de vista microestrutural, alguns efeitos foram observados:

$\checkmark \quad \mathrm{Na}$ característica dos poros: o tamanho de poros aumentou à medida que se elevou a temperatura, o que, provavelmente, deve ter ocorrido pela eliminação da água de constituição presente no minério e nos silicatos. Não ocorreram alterações significativas na porosidade e área total de poros na faixa de temperatura entre 100 e $550^{\circ} \mathrm{C}$ capazes de fragilizar o aglomerado. Isso poderia justificar a tendência da pequena variação da resistência do aglomerado sem exposição à água em função da temperatura de secagem;

$\checkmark \quad \mathrm{Na}$ morfologia do silicato de sódio: em temperaturas de até $200^{\circ} \mathrm{C}$ observouse a formação de filmes homogêneos de silicato de sódio (indicados na Figura 2 por setas contínuas) que são responsáveis pela união entre as partículas de minério de ferro. Entretanto, na faixa de temperaturas entre 200 e $300^{\circ} \mathrm{C}$ verificou-se 0 crescimento de filamentos aciculares sobre esses filmes (indicado na Figura 2 por setas pontilhadas). Acima de $300^{\circ} \mathrm{C}$, esses filamentos se reduzem significativamente. A Figura 2 ilustra a evolução da morfologia da fase ligante no aglomerado em função da temperatura.

* Contribuição técnica ao $44^{\circ}$ Seminário de Redução de Minério de Ferro e Matérias-primas, 15은 Simpósio Brasileiro de Minério de Ferro e 2o Simpósio Brasileiro de Aglomeração de Minério de Ferro, 15 a 18 de setembro de 2014, Belo Horizonte, MG, Brasil. 

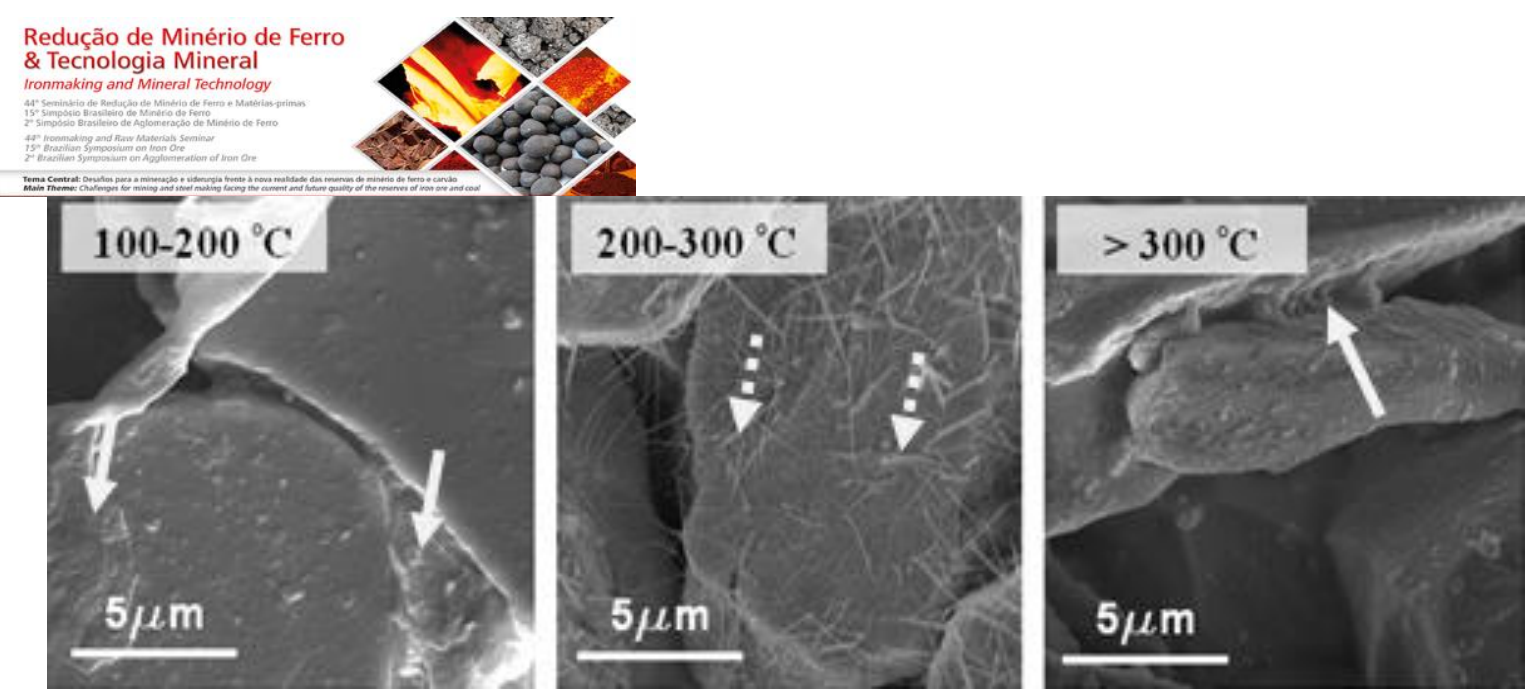

Figura 2. Evolução da morfologia da fase ligante no aglomerado durante secagem/cura com ar atmosférico em função da temperatura.

Com o intuito de identificar a natureza química e cristalográfica dos filamentos aciculares, uma amostra do aglomerado contendo grande quantidade de filamentos aciculares (Figura $3 \mathrm{~A}$ ) foi submetida a análises por difração de raios $\mathrm{X}$ e espectroscopia Raman. A termonatrita $\left(\mathrm{Na}_{2} \mathrm{CO}_{3} \cdot \mathrm{H}_{2} \mathrm{O}\right)$ foi identificada (fase minoritária) na amostra conforme difratograma da Figura 3C. A Figura 3B apresenta o espectro Raman da amostra. Observa-se, a presença de uma banda forte em $1071 \mathrm{~cm}^{-1}$, característica das vibrações de estiramento simétrico presentes no íon $\mathrm{CO}_{3}{ }^{-2}$.

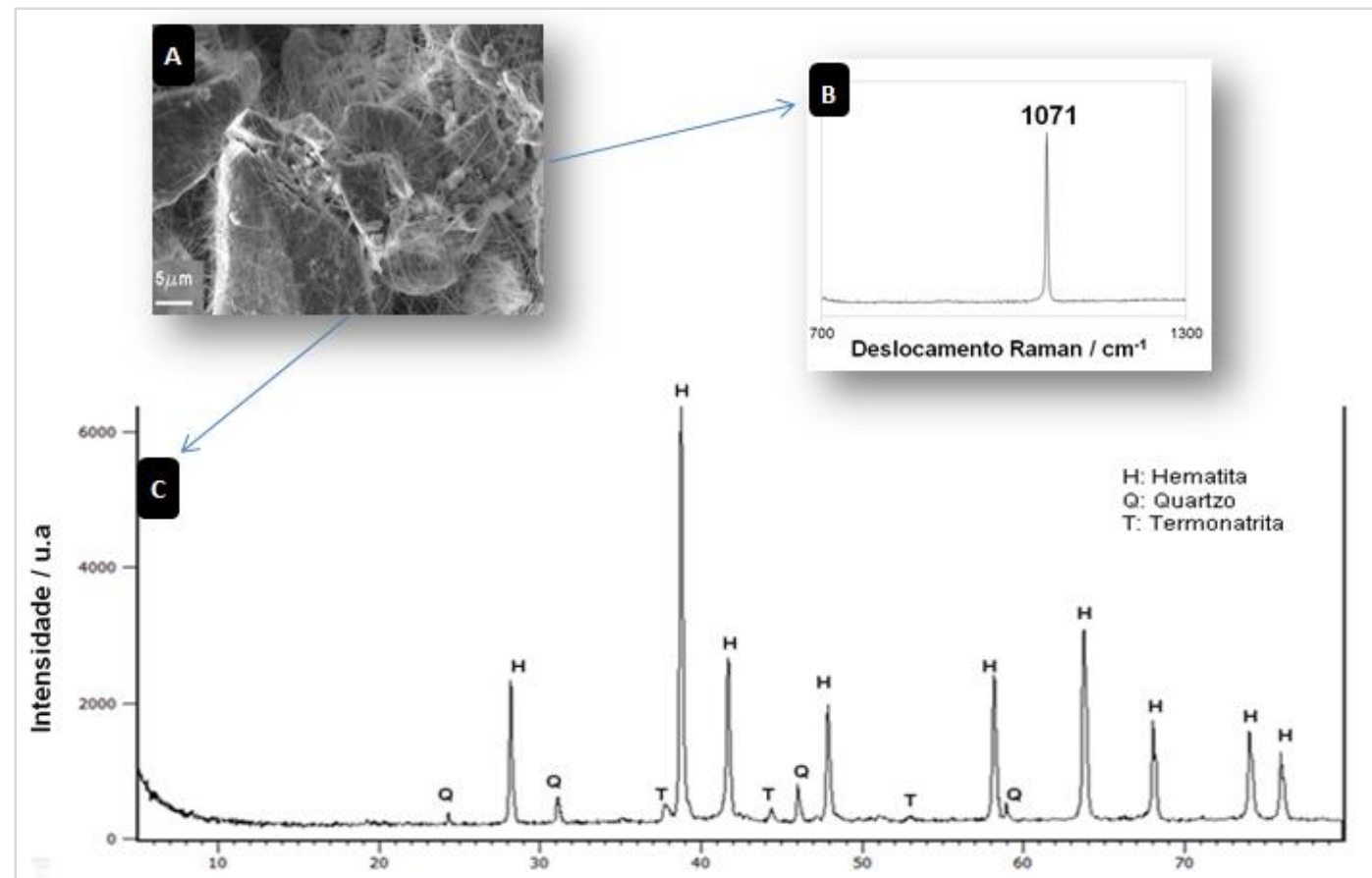

Figura 3. Difratograma de raios $X(C)$ e espectro Raman (B) da amostra contendo filamentos aciculares observados por microscopia eletrônica de varredura $(A)$.

A fim de corroborar a presença da termonatrita na amostra secada a $250^{\circ} \mathrm{C}$, obtiveram-se as curvas de TG/DSC (Figura 4). A curva de TG da amostra mostra claramente dois eventos de perda de massa, parcialmente sobrepostos, entre as temperaturas de $25^{\circ} \mathrm{C}$ e $120^{\circ} \mathrm{C}$. Essas perdas de massa são reveladas na curva de DSC como picos endotérmicos. As temperaturas de ocorrência destes picos são aproximadamente $50^{\circ} \mathrm{C}$ e $92^{\circ} \mathrm{C}$. Em temperaturas mais altas, entre $550^{\circ} \mathrm{C}$ e $810^{\circ} \mathrm{C}$

* Contribuição técnica ao 44 Seminário de Redução de Minério de Ferro e Matérias-primas, $15^{\circ}$ Simpósio Brasileiro de Minério de Ferro e 2o Simpósio Brasileiro de Aglomeração de Minério de Ferro, 15 a 18 de setembro de 2014, Belo Horizonte, MG, Brasil. 
ocorre outro evento de perda de massa de $1,8 \%$. Entretanto, o resultado de DSC nessa região não mostra a presença de pico endotérmico (ou exotérmico), mas apenas um "ombro" largo é notado. De acordo com Seyrankaya [10], o carbonato de sódio mono-hidratado (termonatrita) é convertido em carbonato de sódio anidro pela remoção da água do cristal através de aquecimento em temperaturas acima de $120^{\circ} \mathrm{C}$ ou superior, conforme a reação: $\mathrm{Na}_{2} \mathrm{CO}_{3} \mathrm{H}_{2} \mathrm{O}(\mathrm{s}) \rightarrow \mathrm{Na}_{2} \mathrm{CO}_{3}(\mathrm{~s})+\mathrm{H}_{2} \mathrm{O}(\mathrm{g})$. Deshpande et al. [11] afirmam que a perda de água do carbonato mono-hidratado ocorre em dois estágios $\left(150^{\circ} \mathrm{C}\right.$ e $\left.180^{\circ} \mathrm{C}\right)$, mas que pode ocorrer em temperaturas abaixo de $100^{\circ} \mathrm{C}$ dependendo da natureza e morfologia da amostra. Dessa forma, podem-se atribuir as perdas de massa ocorridas entre 25 a $120^{\circ} \mathrm{C}$ a perda de água combinada do carbonato de sódio mono-hidratado. O outro evento que ocorre entre $550^{\circ} \mathrm{C}$ e $810^{\circ} \mathrm{C}$ está associado à decomposição do carbonato de sódio.

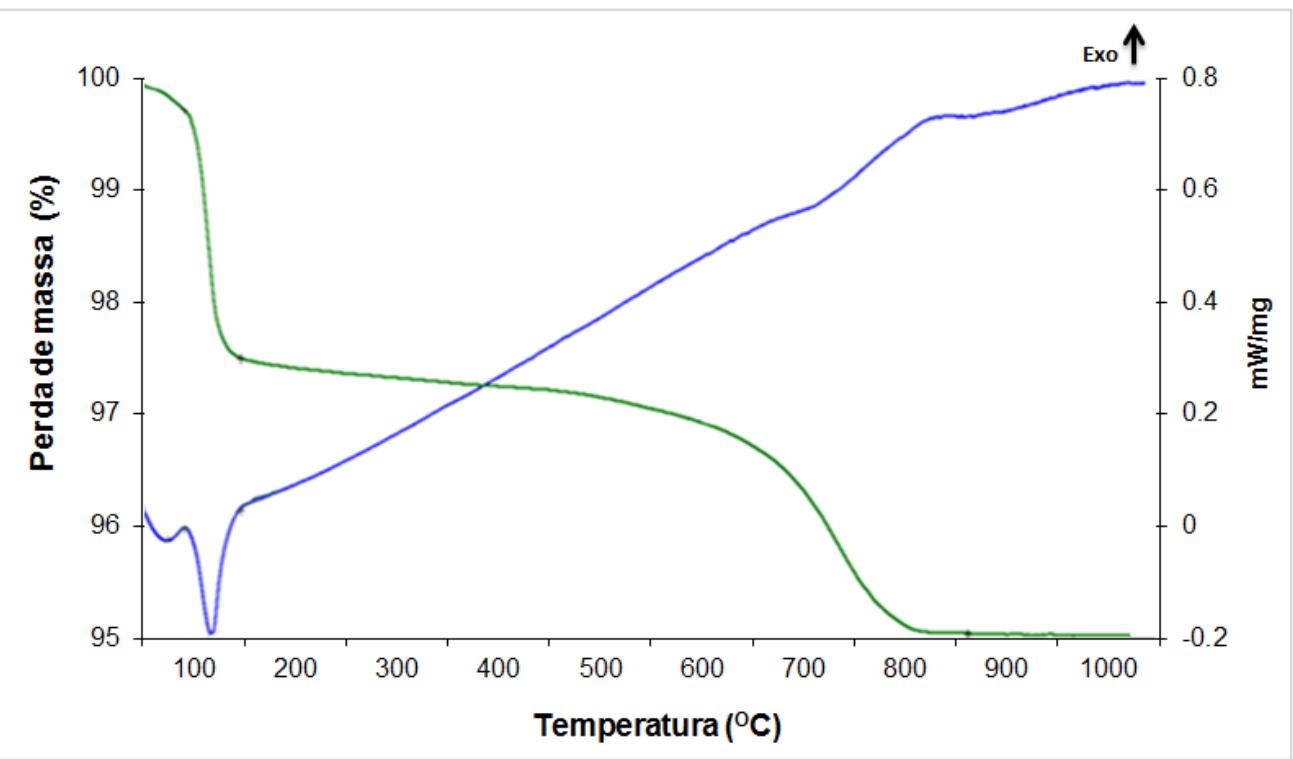

Figura 4. Curva TG/DSC da amostra contendo grande quantidade de filamentos aciculares

Com o objetivo de identificar a solubilidade em água desses filamentos aciculares, uma região característica da amostra seca contendo grande quantidade desses filamentos foi identificada durante observação no MEV. Posteriormente, a amostra foi imersa em água por 5 minutos, secada a $100^{\circ} \mathrm{C}$ e a mesma região observada novamente no MEV. Nota-se a presença maciça desses filamentos antes da imersão e o desaparecimento completo após imersão, constatando assim a solubilidade completa desses filamentos na presença de água (Figura 5).

* Contribuição técnica ao 44 Seminário de Redução de Minério de Ferro e Matérias-primas, 15ํ Simpósio Brasileiro de Minério de Ferro e $2^{\circ}$ Simpósio Brasileiro de Aglomeração de Minério de Ferro, 15 a 18 de setembro de 2014, Belo Horizonte, MG, Brasil. 


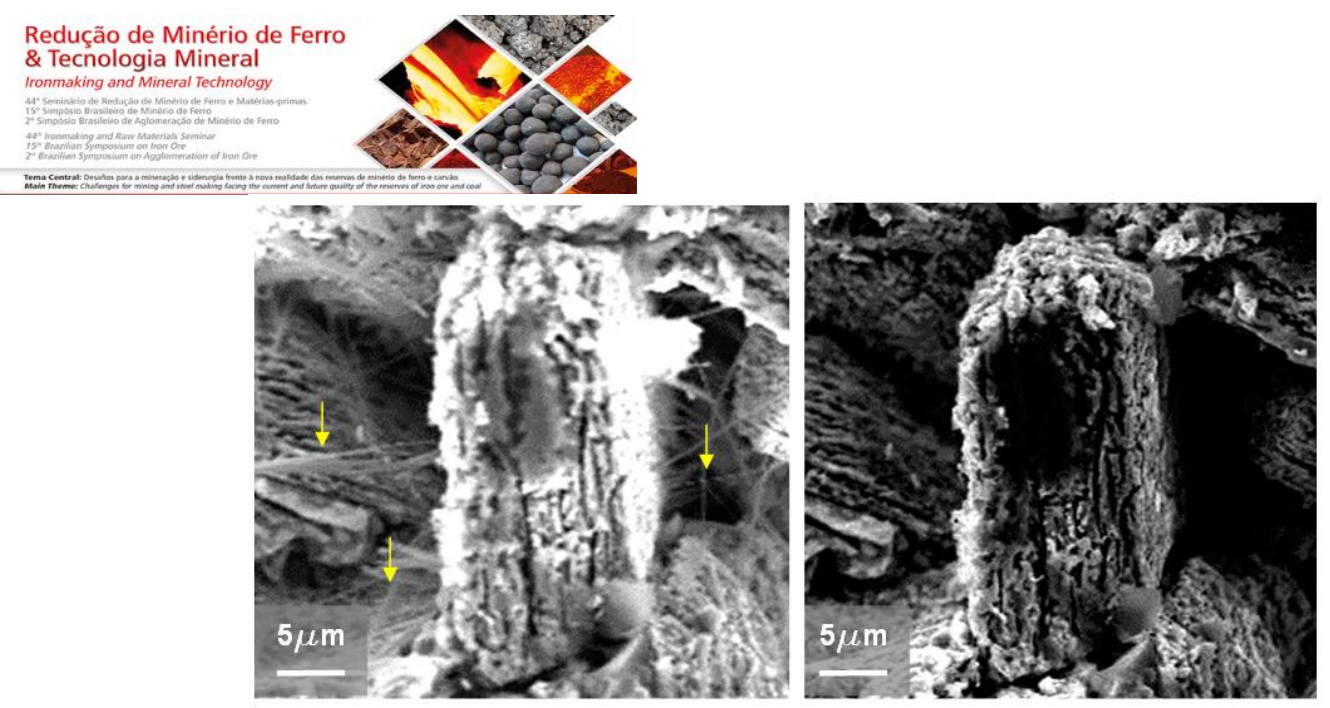

Figura 5. Imagens de MEV da amostra antes (esquerda) e após imersão em água (direita).

Baseado nas constatações dos experimentos realizados, um modelo fenomenológico de secagem do silicato de sódio em um aglomerado de finos de minério de ferro é descrito a seguir (Figura 6 ). A $25^{\circ} \mathrm{C}$, a resistência inicial é provocada pelas forças capilares da água e do silicato de sódio. Nesse estágio, representado pela condição (1) na Figura 6, a solução de silicato de sódio interage com o $\mathrm{CO}_{2}$ presente na atmosfera e inicia o processo de cura e endurecimento, que será acelerado pelas etapas de secagem forçada do processo. Durante o processo de endurecimento e secagem, o silicato de sódio reage com $0 \mathrm{CO}_{2}$ formando carbonato de sódio e sílica gel. Durante o estágio (2), a secagem com ar atmosférico $\left(100^{\circ} \mathrm{C}-150^{\circ} \mathrm{C}\right)$ é responsável pela transformação do gel de silicato em um filme pastoso, ainda muito hidratado. O aquecimento em temperatura mais elevadas, acima de $150^{\circ} \mathrm{C}$, representado pelo estágio (3), promove o crescimento de cristais aciculares de carbonato de sódio mono-hidratado. Esses cristais foram identificados como termonatrita $\left(\mathrm{Na}_{2} \mathrm{CO}_{3} \cdot \mathrm{H}_{2} \mathrm{O}\right)$ e sua presença e quantidade está atribuída à exposição do silicato de sódio ao $\mathrm{CO}_{2}$ e as condições favoráveis para sua nucleação e crescimento. $\mathrm{O}$ aquecimento em temperaturas acima de $350^{\circ} \mathrm{C}$ promove $\mathrm{O}$ desaparecimento dos cristais aciculares de carbonato de sódio mono-hidratado. Em $550^{\circ} \mathrm{C}$, o carbonato de sódio anidro está presente, pois ainda não foi decomposto nessa temperatura. Acima $550^{\circ} \mathrm{C}$, o filme de silicato de sódio encontra-se completamente anidro e com baixa solubilidade em água. $\mathrm{O}$ aquecimento em atmosfera rica em $\mathrm{CO}_{2}(6)$ permite a formação de uma grande quantidade de cristais aciculares de carbonato de sódio mono-hidratado. A lavagem com água dos aglomerados presentes no estágio (3) e (6), solubiliza os cristais e reduz a presença de sódio (5) e (7).

A formação forçada de carbonato de sódio permite a obtenção de filmes com relação em peso $\mathrm{SiO}_{2} / \mathrm{Na}_{2} \mathrm{O}$ mais elevados e com menor solubilidade em água, quando comparados com os filmes provenientes de secagem com apenas ar atmosférico. Além disso, para o mesmo grau de solubilidade, temperaturas de secagem inferiores são requeridas na secagem com a presença de $\mathrm{CO}_{2}$.

* Contribuição técnica ao 44 Seminário de Redução de Minério de Ferro e Matérias-primas, 15o Simpósio Brasileiro de Minério de Ferro e 2o Simpósio Brasileiro de Aglomeração de Minério de Ferro, 15 a 18 de setembro de 2014, Belo Horizonte, MG, Brasil. 

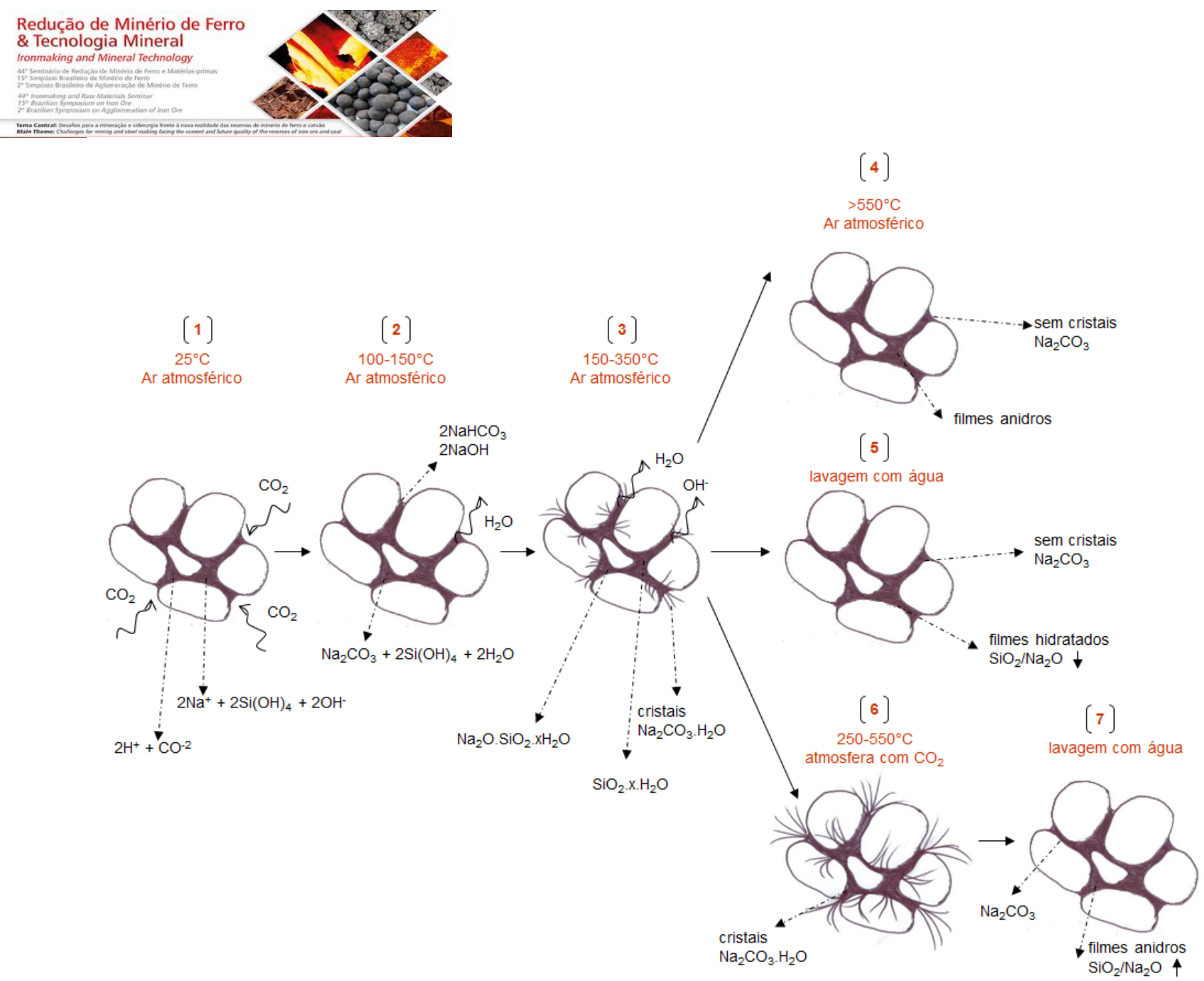

Figura 6. Modelo fenomenológico de secagem e cura do silicato de sódio no aglomerado.

\section{CONCLUSÃo}

A resistência à compressão do aglomerado é influenciada pela temperatura e atmosfera gasosa durante a etapa de secagem e cura. Do ponto de vista macroestrutural não foram notadas alterações na porosidade, formações $e$ propagações de trincas e expansão volumétrica no aglomerado durante a secagem na presença de ar atmosférico até $550^{\circ} \mathrm{C}$ capazes de fragilizar o aglomerado, fato que the confere integridade durante o processo de secagem. Isso poderia justificar a tendência da pequena variação da resistência do aglomerado sem exposição à água em função da temperatura de secagem.

No entanto, do ponto de vista microestrutural, observou-se o crescimento de filamentos aciculares de carbonato de sódio mono-hidratado sobre os filmes de silicato de sódio durante a secagem, em temperaturas entre $200^{\circ} \mathrm{C}$ e $300^{\circ} \mathrm{C}$. Em atmosferas ricas em $\mathrm{CO}_{2}$ observou-se uma formação mais intensa dos filamentos aciculares. Estes filamentos são solúveis em água, formam-se na presença de $\mathrm{CO}_{2} \mathrm{e}$ influenciam a qualidade final do filme de silicato de sódio formado após secagem. $\mathrm{O}$ uso de $\mathrm{CO}_{2}$ durante a secagem do silicato de sódio mostrou-se eficiente para a redução do teor de sódio do filme de silicato (via formação de carbonato de sódio). A menor presença de íons sódio na estrutura do silicato contribui para a formação de filmes com maior grau de polimerização e menor solubilidade.

* Contribuição técnica ao 44 Seminário de Redução de Minério de Ferro e Matérias-primas, 15o Simpósio Brasileiro de Minério de Ferro e 2o Simpósio Brasileiro de Aglomeração de Minério de Ferro, 15 a 18 de setembro de 2014, Belo Horizonte, MG, Brasil. 


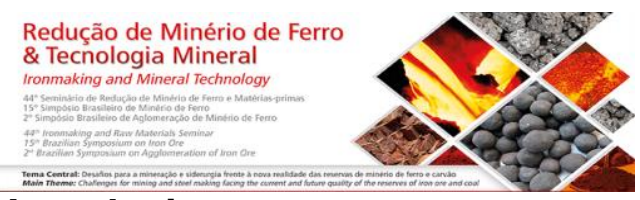

\section{Agradecimentos}

Os autores gostariam de agradecer a toda à equipe envolvida, pelo empenho na execução e interpretação dos resultados, e a Vale S.A, pelo apoio e pela oportunidade de publicar este artigo.

\section{REFERÊNCIAS}

1 VALE S.A (Brasil). Hamilton Porto Pimenta; Flávio de Castro Dutra. Ore Fine Agglomerate to be used in Sintering Process and Production Process of Ore Fines Agglomerate. WO/2011/061627. 26 May. 2011.

2 Owusu YA. Physical-chemistry study of sodium silicate as a foundry sand binder. Advances in Colloid and Interface Science, Detroit, v. 18, p. 57-91, 1982.

3 De Boer JH, Hermans MEA, Vleesken M. Chemisorption and physical adsorption of water on $\mathrm{SiO}_{2}$ surface. Konink Ned. Akad. Proc., s.I, 1958; 60( 45-49): 234-244.

4 Dulag R, Rosenbaum J. Industrial Cold Adhesives: A Practical Handbook for the Maker and User. London: Charles Griffin \& Company, Limited, 1937.

$5 \quad$ Iller RK. The chemistry of silica: solubility, polymerization, colloid and surface properties and biochemistry. New York: J. Wiley \& Sons, 1979. (Cap. 1-The Occurrence, Dissolution, and Deposition of Silica; Cap. 2-Polymerization of Silica; Cap. 3-Water-Soluble Silicates).

6 Lucas S, Tognonvi MT, Gelet J-L, Soro J, Rossignol S. Interactions between silica sand and sodium silicate solution during consolidation process. Journal of NonCrystalline Solids, s.I, 2011; 357: 1310-1318.

$7 \mathrm{Kim} \mathrm{EH}$, Lee JH, Jung YG, Jang JC, Paik U. Control of $\mathrm{H}_{2} \mathrm{O}$ generated during the $\mathrm{CO}_{2}$ hardening process in a casting mold. Ceramics International, s.l, 2013; 39: 3393-3998.

8 Tognonvi MT, Soro J, Rossignol S. Physico-chemistry of silica/alkaline silicate interactions during consolidation. Part 1: Effect of cation size. Journal of NonCrystalline Solids, s.I, 2012; 358: 81-87.

9 Tognonvi MT, Soro J, Gelet JL, Rossignol S. Physico-chemistry of silica/Na silicate interactions during consolidation. Part 2: Effect of $\mathrm{pH}$. Journal of Non-Crystalline Solids, s.I, 2012; 358(3): 492-501.

10 Seyrankaya A, Ozalp B. Dehydration of sodium carbonate monohydrate with indirect microwave heating. Thermochimica Acta, s.l, 2006; 448: 31-36.

11 Deshpande DA, Ghormare KR, Jawadekar VL, Deshpande ND. Thermal Analysis of $\mathrm{Na}_{2} \mathrm{CO}_{3}-\mathrm{H}_{2} \mathrm{O}$ Crystals. Thermochimica Acta, 1983; 60: 295-30.

* Contribuição técnica ao 44 Seminário de Redução de Minério de Ferro e Matérias-primas, 15ㅇ Simpósio Brasileiro de Minério de Ferro e 2o Simpósio Brasileiro de Aglomeração de Minério de Ferro, 15 a 18 de setembro de 2014, Belo Horizonte, MG, Brasil. 